\section{Adaptação transcultural para o português (Brasil) da Vulnerability to Abuse Screening Scale (VASS) para rastreio da violência contra idosos}

\author{
Brazilian Portuguese cross-cultural adaptation of \\ the Vulnerability to Abuse Screening Scale (VASS) \\ for screening of violence against the elderly
}

\author{
Adaptación transcultural para el Portugués (Brasil) \\ de la Vulnerability to Abuse Screening Scale (VASS) \\ para la detección de la violencia contra ancianos
}

\author{
${ }_{1}$ Grupo de Estudos Psicologia \\ e Saúde, Universidade \\ Federal do Rio Grande do \\ Norte, Natal, Brasil. \\ Correspondência \\ R. S. Maia \\ Grupo de Estudos Psicologia \\ e Saúde, Departamento de \\ Psicologia, Universidade \\ Federal do Rio Grande \\ do Norte. \\ Rua Humberto Monte 1756 \\ Natal, RN 59082-190, Brasil. \\ rodrigo_maia89@yahoo.com.br
}

\section{Abstract}

The literature indicates a lack of available tools in Portuguese for detecting violence against the elderly. It thus seems appropriate to provide Portuguese-language versions of tools originating from other cultures. The Vulnerability to Abuse Screening Scale (VASS) contains 12 items that identify the risk of violence against the elderly. The current study aimed to conduct a Brazilian Portuguese cultural adaptation of VASS. The concept used to construct the instrument and its items proved appropriate for investigating the target phenomenon. The study showed good semantic equivalence between items in the back-translations and the original instrument, especially for $T 1$ - R1. The judges opted to use 11 items from $T 1$ for the short version. Operational equivalence was satisfactory. The results were generally acceptable. However, the instrument still failed to show sufficiently satisfactory results to confirm the feasibility of its use. In conclusion, the instrument needs to be revised and replicated to further verify its validity.

Domestic Violence; Elder Abuse; Aged; Questionnaires
Rodrigo da Silva Maia 1

Eulália Maria Chaves Maia 1

\section{Resumo}

A literatura aponta a insuficiência de instrumentos disponibilizados em português para a detecção da violência contra idosos. Assim, parece oportuno disponibilizar versões lusófonas de ferramentas advindas de outras culturas. A Vulnerability to Abuse Screening Scale (VASS) contém 12 itens que constatam o risco à violência contra idosos. Este trabalho objetivou promover a adaptação transcultural para o Brasil da VASS. Verificou-se que o conceito utilizado para a construção do instrumento, bem como seus itens mostram-se adequados à investigação do fenômeno. Evidenciou-se boa equivalência semântica entre os itens das retrotraduções e do instrumento original, especialmente quanto aos resultados de $T 1-R 1$. Os juízes optaram pelo uso de 11 itens de T1 à versão-síntese. A equivalência operacional mostrou-se satisfatória. Em geral, os resultados apresentados mostram-se aceitáveis. Destaca-se que o instrumento ainda não apresentou resultados satisfatórios que indiquem viabilidade de seu uso. Conclui-se que é necessário revisar, replicar o instrumento para a verificação da validade.

Violência Doméstica; Maus-Tratos ao Idoso; Idoso; Questionários 
Introdução

O envelhecimento populacional é um fenômeno vivenciado mundialmente. No Brasil, esse fenômeno ocorre vertiginosamente, prevendo-se que representaremos a sexta maior população idosa no mundo 1 . Associado ao envelhecimento populacional existe a necessidade de desenvolver políticas públicas voltadas à atenção integral do idoso 2. No entanto, sabe-se que a atenção à pessoa idosa não vem sendo garantida, especialmente em decorrência de contextos de maustratos e violências contra essa população.

A violência contra o idoso é um desafio às políticas sociais, uma vez que não envolve somente o idoso vítima, mas também sua família, os profissionais que dele cuidam e todo o sistema de proteção/garantia dos direitos da pessoa idosa. A identificação do idoso vítima da violência ainda é precária e como principais motivos da interdição da informação, dificuldade de identificação e à subnotificação da violência, destacam-se o grau de proximidade do agressor e as relações de dependência afetivo-emocional, de cuidado ou financeira que existem entre vítima/agressor 3,4.

A literatura destaca que é perceptível a insuficiência de instrumentos disponibilizados em português para a detecção desse fenômeno $5,6,7$. Diante disso, parece oportuno disponibilizar versões lusófonas de ferramentas advindas de investigações consistentes e bem estabelecidas para outras culturas. A Vulnerability to Abuse Screening Scale (VASS), desenvolvida na realidade australiana 8,9 , foi escolhida para ser objeto do estudo pretendido. A escala, de autorrelato a ser respondida pelo idoso e aplicável em contextos clínicos e domiciliares, contém doze itens que constatam o risco à violência doméstica contra o idoso com base em quatro domínios, a saber: Físico, Psicológico, Negligência e Financeiro.

Segundo os autores do estudo australiano, o instrumento tem o potencial de auxiliar no desenvolvimento, operacionalização e consolidação de políticas públicas de prevenção, na triagem e na intervenção defronte ao fenômeno da violência ${ }^{8,9}$. Diante do exposto, o presente trabalho teve como objetivo promover a adaptação transcultural para o Brasil da VASS. Especificamente, pretendeu-se: (a) realizar a equivalência conceitual e de itens entre o instrumento original e a versão brasileira; (b) realizar a equivalência semântica, especialmente os aspectos idiomáticos (literais) e culturais; (c) proceder a equivalência operacional.

\section{Método}

\section{Desenho e procedimentos}

Os passos para a adaptação transcultural seguirão a proposta de operacionalização calcada na apreciação de diferentes tipos de equivalência 10: equivalência conceitual e de itens, equivalência semântica e equivalência operacional. Para alcançar a equivalência conceitual e de itens e a etapa de equivalência semântica, utilizou-se a técnica do painel de especialistas 11. A técnica empregada consiste em reunir um grupo de $e x$ pertises, habilitados e conhecedores da temática, que possam contribuir com a discussão sobre a construção, adaptação e validação do instrumento que se pretende investir. Já quanto à etapa da equivalência operacional, participaram idosos não institucionalizados e sem comprometimento cognitivo, frequentadores de um programa de promoção à saúde de uma unidade do Programa Saúde da Família e de uma instituição de saúde privada, de um município de grande porte do nordeste brasileiro.

\section{Equivalência conceitual e dvvte itens}

Nesta etapa, o instrumento foi apreciado por três expertises na área de violência, desenvolvimento humano e/ou gerontologia, além de conhecedores dos métodos de adaptação transcultural, os quais avaliaram o conceito base utilizado e a pertinência dos itens do instrumento original para a captação do fenômeno estudado. Esses especialistas indicariam a adequabilidade do conceito e dos itens para investigar o fenômeno que se pretende aferir na realidade do país-destino à adaptação transcultural.

\section{Equivalência semântica}

Na etapa de equivalência semântica, inicialmente, o instrumento sofreu duas traduções do original em língua inglesa à portuguesa (T1 e T2). Em seguida, o instrumento foi retrotraduzido por outros dois tradutores (R1 e R2) para a língua original. Ambas as etapas foram realizadas de forma independente, cegas e paralelas, com profissionais formados em letras, atuantes como tradutores e, ainda que nascidos no Brasil, fluentes na língua materna do instrumento. A partir de então, os expertises foram convidados a avaliar semântica e literalmente (equivalências idiomática e cultural) as retrotraduções com o instrumento original. Por fim, procedeu-se a escolha dos itens, por meio de consenso, à elaboração da versão síntese. 


\section{Equivalência operacional}

A versão síntese do instrumento foi dirigida a juízes não-especialistas. Portanto, convidou-se um total de 15 idosos, aos quais foi solicitada a avaliação do instrumento para verificar a sua adequabilidade em termos de compreensão e pertinência de seus itens, objetivando elaborar a versão síntese final a ser testada numa população maior. Os idosos convidados tinham diferentes níveis de escolaridade e condições socioeconômicas.

\section{Análise dos dados}

Para a avaliação dos juízes do painel de especialistas, bem como dos juízes não-especialistas, utilizou-se o kappa de Fleiss (k), que verifica a confiabilidade do acordo na avaliação entre juízes. A interpretação do coeficiente kappa pode ser classificada como: (a) concordância quase perfeita, para valores entre 0,81 e 1,00; (b) concordância substancial, quando entre 0,61 e 0,80 ; (c) concordância moderada entre 0,41 e 0,60 ; (d) concordância fraca ou pequena entre $0,21 \mathrm{e}$ 0,40 ; (e) concordância leve entre 0,0 e 0,20; e (f) nenhuma correlação quando forem menores do que zero (0), indicando ausência de acordo ${ }^{12}$. Os demais dados foram analisados usando-se estatística descritiva. As análises foram realizadas no programa Minitab 15.1 (Minitab Inc., State College, Estados Unidos).

\section{Aspectos éticos}

A pesquisa foi aprovada pelo Comitê de Ética em Pesquisa com Seres Humanos de uma instituição de ensino superior pública, sob parecer de o 97.186/2012 (CONEP).

\section{Resultados e discussão}

Com relação à equivalência conceitual e de itens, o grupo de especialistas, alicerçados por consultas a referências bibliográficas acerca do tema, apontaram que os conceitos relacionados à violência doméstica contra idosos empregados para a elaboração da VASS eram pertinentes ao contexto cultural brasileiro, especialmente em relação às populações urbanas. Um dos juízes destacou que o item 4 da escala original poderia não ser bem compreendido na realidade de uma população de baixa renda, pois " a aplicabilidade do termo 'privacidade', em uma residência em que vivem cinco pessoas e que tenha apenas um cômodo pode gerar confusão nas respostas, por exemplo" (sic). Entretanto, o mesmo indicou que se observasse, durante a etapa de pré-teste, se isso ocorreria.

No que tange à etapa de equivalência semântica, evidenciou-se uma boa equivalência entre os itens oriundos das retrotraduções e os do instrumento original, especialmente quando se referem aos resultados referentes à díade $\mathrm{T} 1-\mathrm{R} 1$ (Tabela 1). Grosso modo, os juízes, por meio de consenso, optaram pelo uso de itens oriundos de T1 (11 itens) para a elaboração da versãosíntese, seja incorporando-os de forma integral ou parcialmente após modificações, conforme demonstra a Tabela 2 .

A Tabela 2 mostra ainda as modificações realizadas durante a equivalência semântica, que contemplaram os itens 2, 6, 7 e 9. Percebe-se que a maioria das alterações pretendeu tornar a versão em português mais coloquial e aceitável para a população-destino. A concordância das avaliações empreendidas durante a equivalência semântica, verificada com o auxílio do kappa de Fleiss foi de 0,244 , o que indica concordância fraca ou pequena 12 . Ainda assim, os expertises sugeriram, consensualmente, a submissão do instrumento ao pré-teste objetivando verificar o comportamento dos respondentes não-especialistas.

$\mathrm{Na}$ etapa de equivalência operacional, participaram 15 idosos com idades entre 62 e 82 anos (72,2; desvio-padrão $=7,28)$, sendo 6 do sexo masculino e 9 do feminino, e com diferentes níveis de escolaridade: baixo: Ensino Fundamental incompleto/completo; médio: Ensino Médio incompleto/completo; alto: Superior e/ou pósgraduação incompleto/completo, em que registramos cinco sujeitos em cada categoria. Os idosos participantes avaliaram o instrumento como adequado e compreensível à população a que se destina, observado no acordo um valor bruto de $92,5 \%$. A concordância, verificada com o auxílio do kappa de Fleiss foi de 0,585 , o que indica concordância moderada 12 . As inadequações podem ser observadas na Tabela 3. Apesar das inadequações serem significativas, consultou-se o grupo de especialistas, que sugeriram manter a redação original dos itens.

Salienta-se que valores de confiabilidade moderados foram encontrados também no estudo inicial das propriedades do instrumento ${ }^{8}$. Destaca-se que o instrumento não apresentou resultados satisfatórios que indiquem a viabilidade de seu uso. Diante disso, faz-se necessário ressubmetê-lo à revisão pelos juízes especialistas 11 e, posteriormente, reaplicação do instrumento entre os juízes não-especialistas, para a verificação da concordância e evidências de validade 10 . 
Comparação entre o original em inglês e as retrotraduções do instrumento Vulnerability to Abuse Screening Scale (VASS).

\begin{tabular}{|c|c|c|c|c|c|c|}
\hline Original & $\mathrm{T} 1 \rightarrow \mathrm{R} 1$ & I & C & $\mathrm{T} 2 \rightarrow \mathrm{R} 2$ & I & C \\
\hline $\begin{array}{l}\text { 1. Are you afraid of anyone in your } \\
\text { family? }\end{array}$ & $\begin{array}{l}\text { 1. Are you afraid of anyone in your } \\
\text { family? }\end{array}$ & 05 * & $\mathrm{IN}$ * & $\begin{array}{l}\text { 1. Are you afraid of anyone in your } \\
\text { family? }\end{array}$ & 05 * & IN * \\
\hline $\begin{array}{l}\text { 2. Has anyone close to you tried to hurt } \\
\text { you or harm you recently? }\end{array}$ & $\begin{array}{l}\text { 2. Has anyone close to you tried to hurt } \\
\text { you or harm you recently? }\end{array}$ & 05 * & PA & $\begin{array}{l}\text { 2. Has anyone close tried to hurt or } \\
\text { mistreat you recently? }\end{array}$ & 02 & PA \\
\hline $\begin{array}{l}\text { 3. Has anyone close to you called you } \\
\text { names or put you down or made you } \\
\text { feel bad recently? }\end{array}$ & $\begin{array}{l}\text { 3. Has anyone close to you called you } \\
\text { names or put you down or made you feel } \\
\text { bad recently? }\end{array}$ & 05 * & IN & $\begin{array}{l}\text { 3. Has anyone close to you verbally } \\
\text { offended you, put you down or made } \\
\text { you feel bad recently? }\end{array}$ & 03 & PA \\
\hline $\begin{array}{l}\text { 4. Do you have enough privacy at } \\
\text { home? }\end{array}$ & 4. Do you have enough privacy at home? & 05 * & IN & $\begin{array}{l}\text { 4. Do you have enough privacy inside } \\
\text { your home? }\end{array}$ & 05 & PA \\
\hline $\begin{array}{l}\text { 5. Do you trust most of the people in } \\
\text { your family? }\end{array}$ & $\begin{array}{l}\text { 5. Do you trust most of the people in } \\
\text { your family? }\end{array}$ & 05 * & IN & $\begin{array}{l}\text { 5. Do you trust most of the people in } \\
\text { your family? }\end{array}$ & 05 & PA \\
\hline $\begin{array}{l}\text { 6. Can you take your own medication } \\
\text { and get around by yourself? }\end{array}$ & $\begin{array}{l}\text { 6. Can you take your own medication and } \\
\text { get around by yourself? }\end{array}$ & 05 * & $\mathrm{PA}$ * & $\begin{array}{l}\text { 6. Can you control your own } \\
\text { medication and take care of you on } \\
\text { your own? }\end{array}$ & 02 * & PA \\
\hline $\begin{array}{l}\text { 8. Do you feel that nobody wants you } \\
\text { around? }\end{array}$ & $\begin{array}{l}\text { 8. Do you feel that nobody wants you } \\
\text { around? }\end{array}$ & 05 * & IN & $\begin{array}{l}\text { 8. Do you feel that no one wants you } \\
\text { around? }\end{array}$ & 04 & IN \\
\hline $\begin{array}{l}\text { 9. Do you feel uncomfortable with } \\
\text { anyone in your family? }\end{array}$ & $\begin{array}{l}\text { 9. Do you feel uncomfortable with } \\
\text { anyone in your family? }\end{array}$ & 05 * & IN & $\begin{array}{l}\text { 9. Do you feel uncomfortable with } \\
\text { anyone from your family? }\end{array}$ & 05 & PA \\
\hline $\begin{array}{l}\text { 10. Does someone in your family make } \\
\text { you stay in bed or tell you you're sick } \\
\text { when you know you're not? }\end{array}$ & $\begin{array}{l}\text { 10. Does someone in your family make } \\
\text { you stay in bed or tell you you're sick } \\
\text { when you know you're not? }\end{array}$ & 05 * & IN & $\begin{array}{l}\text { 10. Does anyone in your family make } \\
\text { you stay in bed or say that you're sick } \\
\text { when know you're not? }\end{array}$ & 05 * & PA \\
\hline $\begin{array}{l}\text { 11. Has anyone forced you to do things } \\
\text { you didn't want to do? }\end{array}$ & $\begin{array}{l}\text { 11. Has anyone forced you to do things } \\
\text { you didn't want to do? }\end{array}$ & 05 * & IN & $\begin{array}{l}\text { 11. Has anyone forced you to do things } \\
\text { you didn't want to do? }\end{array}$ & 05 * & PA \\
\hline $\begin{array}{l}\text { 12. Has anyone taken things that } \\
\text { belong to you without your OK? }\end{array}$ & $\begin{array}{l}\text { 12. Has anyone taken things that belong } \\
\text { to you without your OK? }\end{array}$ & 05 * & $\mathrm{IN}$ * & $\begin{array}{l}\text { 12. Has anyone taken things } \\
\text { that belong to you without your } \\
\text { permission? }\end{array}$ & 05 & IN \\
\hline
\end{tabular}

C: resultado médio do significado geral (equivalência cultural): IN: inalterado, PA: pouco alterado, MA: muito alterado; I: resultado médio do significado referencial (equivalência idiomática): 01 = nenhum referencial denotativo, 02 = baixo referencial denotativo, 03 = nem baixo e nem alto referencial denotativo, 04 = alto referencial denotativo, 05 = total referencial denotativo; R1: primeira retrotradução; T2: segunda tradução do inglês para o português; R2: segunda retrotradução; T1: primeira tradução do inglês para o português.

* Item unânime na avaliação dos especialistas.

\section{Conclusões}

Os resultados das subetapas apresentadas da adaptação transcultural da VASS mostraram-se satisfatórios, ainda que não conclusivos quanto à sua validade, mas indicam continuidade às etapas subsequentes. Destaca-se que ainda há de desenvolver-se a etapa concernente à devolução dos resultados obtidos no pré-teste para avaliação dos juízes especialistas, a fim de verificar posteriormente as propriedades psicométricas preliminares do instrumento, tais como: (a) equivalência de mensuração entre o instrumento original e o adaptado para a cultura destino; e (b) evidências de fidedignidade e de validade, de constructo, de conteúdo, concorrente, por exemplo. 
Versão síntese do Vulnerability to Abuse Screening Scale (VASS)

\section{Item escolhido}

1. Você tem medo de alguém em sua família?

2. Alguém próximo a você tentou lhe machucar ou prejudicar?

3. Alguém próximo a você lhe ofendeu, colocou para baixo ou o (a) fez sentir-se mal recentemente?

4. Você tem privacidade suficiente em casa?

5. Você confia na maioria das pessoas de sua família?

6. Você consegue tomar suas próprias medicações e locomover-se sozinho (a)?

7. Você fica triste ou solitário (a) com frequência?

8. Você sente que ninguém o (a) quer por perto?

9. Você se sente desconfortável com alguém de sua família?

10. Alguém de sua família faz você ficar na cama ou the diz que você está doente, quando você sabe que não está?

11. Alguém forçou você a fazer coisas que não queria fazer?

12. Alguém tem pego coisas que lhe pertencem sem sua permissão?
Origem

Item modificado

T1: item oriundo da primeira tradução; T2: item oriundo da segunda tradução.

* Item unânime na avaliação dos especialistas;

** Item alterado na avaliação interjuízes.

Itens avaliados inadequados e a justificativa, seguida da sugestão de alteração.

\begin{tabular}{|c|c|c|}
\hline Item inadequado * & $\mathbf{N} \star \star$ & 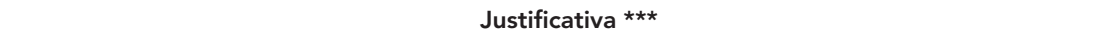 \\
\hline $\begin{array}{l}\text { 2. Alguém próximo a você tentou lhe } \\
\text { machucar ou prejudicar? }\end{array}$ & 2 & $\begin{array}{l}\text { Inadequação única: Em razão dos itens em questão não referirem-se ao familiar ou à família, uma } \\
\text { vez que na reflexão dos mesmos, o instrumento parece investigar violência intrafamiliar. Sugeriu-se } \\
\text { substituir "Alguém próximo a você" por "Algum familiar seu". }\end{array}$ \\
\hline $\begin{array}{l}\text { 3. Alguém próximo a você lhe ofendeu, } \\
\text { colocou para baixo ou o (a) fez sentir-se } \\
\text { mal recentemente? }\end{array}$ & 2 & $\begin{array}{l}\text { 1ạ Inadequação: Mesma inadequação sugerida ao item no } 2 \text {. } \\
\text { 2a Inadequação: Sobre a falta de clareza do sentido atribuído ao "colocar para baixo" e/ou ao de } \\
\text { "fazer mal". }\end{array}$ \\
\hline $\begin{array}{l}\text { 6. Você consegue tomar suas próprias } \\
\text { medicações e locomover-se sozinho (a)? }\end{array}$ & 1 & $\begin{array}{l}\text { Inadequação única: O item contemplar duas perguntas em uma, podendo comprometer a } \\
\text { compreensão dos respondentes. Sugeriu-se o desmembramento em dois itens: "Você consegue } \\
\text { tomar suas próprias medicações?" e "Você consegue andar sozinho (a)?". }\end{array}$ \\
\hline $\begin{array}{l}\text { 10. Alguém de sua família faz você ficar } \\
\text { na cama ou lhe diz que você está doente, } \\
\text { quando você sabe que não está? }\end{array}$ & 1 & $\begin{array}{l}\text { 1ạ Inadequação: Semelhante a do item no 6, sugerindo a criação de duas questões: "Alguém de sua } \\
\text { família faz você ficar na cama, quando você sabe que não precisa estar?" e "Alguém de sua família } \\
\text { lhe diz que você está doente, quando você sabe que não está?". } \\
\text { 2a Inadequação: Sobre o sentido atribuído ao termo "ficar de cama", o qual pode ser atribuído pelo } \\
\text { respondente como uma postura de cuidado por parte dos familiares. }\end{array}$ \\
\hline $\begin{array}{l}\text { 11. Alguém forçou você a fazer coisas que } \\
\text { não queria fazer? }\end{array}$ & 2 & $\begin{array}{l}\text { Inadequação única: Mesma inadequação sugerida ao item no 2. Sugeriu-se substituir "Alguém } \\
\text { forçou" por "Alguém de sua família já forçou". }\end{array}$ \\
\hline $\begin{array}{l}\text { 12. Alguém tem pego coisas que lhe } \\
\text { pertencem sem sua permissão? }\end{array}$ & 2 & $\begin{array}{l}\text { Inadequação única: Mesma inadequação sugerida ao item no } 2 \text {. Sugeriu-se substituir "Alguém tem } \\
\text { pego" por "Alguém de sua família tem pego". }\end{array}$ \\
\hline
\end{tabular}

* Item avaliado como inadequado pelos idosos participantes da etapa de equivalência operacional;

** Número de respondentes que avaliou o item como inadequado;

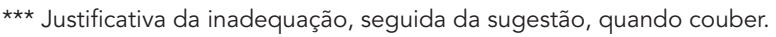




\section{Resumen}

La literatura señala la insuficiencia de instrumentos que estén disponibles en portugués para la detección de la violencia contra la tercera edad. Por ello, parece oportuno poner a disposición del público interesado versiones lusófonas de herramientas que provengan de otras culturas. La Vulnerability to Abuse Screening Scale (VASS) contiene 12 ítems que constatan el riesgo de la violencia contra la tercera edad. Este trabajo tuvo como meta promover la adaptación transcultural para Brasil de la VASS. Se verificó el concepto utilizado para la construcción de este instrumento, así como que sus items se mostraran adecuados para la investigación del fenómeno. Se evidenció una buena equivalencia semántica entre los ítems de las retrotraducciones y del instrumento original, especialmente, en cuanto a los resultados de T1 - R1. Los jueces optaron por el uso de 11 items de T1 en la versión-síntesis. La equivalencia operacional se mostró satisfactoria. En general, los resultados presentados se muestran aceptables. Se destaca que el instrumento todavía no presentó resultados satisfactorios que indiquen la viabilidad de su uso. Se concluye que es necesario revisar, replicar el instrumento con el fin de verificar su validez.

Violencia Doméstica; Maltrato al Anciano; Anciano; Cuestionarios

\section{Colaboradores}

R. S. Maia foi o coordenador da coleta dos dados, conduziu a análise e processamento dos dados e participou da elaboração do artigo. E. M. C. Maia participou do desenvolvimento teórico-metodológico e colaborou na redação do manuscrito.

\section{Referências}

1. Lima-Costa MF, Veras R. Saúde pública e envelhecimento. Cad Saúde Pública 2003; 19:700-1.

2. Veras R. Envelhecimento populacional contemporâneo: demandas, desafios e inovações. Rev Saúde Pública 2009; 43:548-54.

3. Minayo MCS. Violência contra idosos: relevância para um velho problema. Cad Saúde Pública 2003; 19:783-91.

4. Wanderbroocke ACNS, Moré CLOO. Abordagem profissional da violência familiar contra o idoso em uma unidade básica de saúde. Cad Saúde Pública 2013; 29:2513-22.

5. Paixão Jr. CM, Reichenheim ME. Uma revisão sobre instrumentos de rastreamento de violência doméstica contra o idoso. Cad Saúde Pública 2006; 22:1137-49.

6. Paixão Jr. CM, Reichenheim ME, Moraes CL, Coutinho ESF, Veras RP. Adaptação transcultural para o Brasil do instrumento Caregiver Abuse Screen (CASE) para detecção de violência de cuidadores contra idosos. Cad Saúde Pública 2007; 23:2013-22.

7. Reichenheim ME, Paixão Jr. CM, Moraes CL. Adaptação transcultural para o português (Brasil) do instrumento Hwalek-Sengstock Elder Abuse Screening Test (H-S/EAST) utilizado para identificar risco de violência contra o idoso. Cad Saúde Pública 2008; 24:1801-13.
8. Schofield MJ, Reynolds R, Mishra GD, Powers JR, Dobson AJ. Screening for vulnerability to abuse among older women: Women's Health Australia Study. J Appl Gerontol 2002; 21:24-39.

9. Schofield MJ, Mishra GD. Validity of self-report screening scale for elder abuse: Women's Health Australia Study. Gerontologist 2003; 43:110-20.

10. Reichenheim ME, Moraes CL. Operacionalização de adaptação transcultural de instrumentos de aferição usados em epidemiologia. Rev Saúde Pública 2007; 41:665-73.

11. Pinheiro JQ, Farias TM, Abe-Lima JY. Painel de especialistas e estratégia multimétodos: reflexões, exemplos, perspectivas. Psico (Porto Alegre) 2013; 44:184-92.

12. Landis JR, Koch GG. The measurement of observer agreement for categorical data. Biometrics 1977; 33:159-74.

Recebido em 29/Jan/2014

Versão final respresentada em 6/Mar/2014

Aprovado em 28/Abr/2014 